\title{
Magnetic and Ferroelectric Ordering in the $\mathrm{TbMnO}_{3}$ Film
}

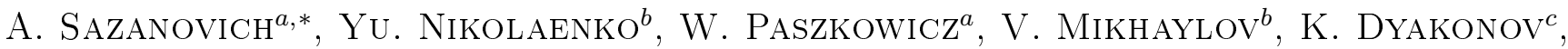 \\ Yu. MedvedeV ${ }^{b}$, V. NIZHANKOVSKII ${ }^{d}$, V. DYAKONOV ${ }^{a, b}$ AND H. SZYMCZAK ${ }^{a}$ \\ ${ }^{a}$ Institute of Physics, PAS, al. Lotników 32/46, 02-668 Warsaw, Poland \\ ${ }^{b}$ A.A. Galkin Donetsk Physico-Technical Institute, NANU, 83114 Donetsk, R. Luxembourg str. 72, Ukraine \\ ${ }^{c}$ A.F. Ioffe Physico-Technical Institute RAN, Politekhnicheskaja 26, 194021 St.-Petersburg, Russia \\ ${ }^{d}$ International Laboratory of High Magnetic Fields and Low Temperatures, 53-421 Wrocław, Poland
}

(Received October 25, 2013; in final form December 9, 2013)

\begin{abstract}
Measurements of magnetization and electric polarization performed for the $\mathrm{TbMnO}_{3}$ film grown onto the single crystal [100] $\mathrm{SrTiO}_{3}$ substrate using magnetron sputtering technique exhibit series of anomalies related to the magnetic and electric ordering of the $\mathrm{Tb}^{3+}$ and $\mathrm{Mn}^{3+}$ sublattices. The detailed temperature dependences of the electric polarization and dielectric constant of the $\mathrm{TbMnO}_{3}$ film have shown that the ferroelectric phase appears below $30 \mathrm{~K}$ in magnetic field $H>1 \mathrm{~T}$ applied in-plane and out-of-plane of the film.
\end{abstract}

DOI: 10.12693/APhysPolA.125.128

PACS 75.47.Lx, 75.85.+t, 75.70.Ak, 77.55.Nv

\section{Introduction}

Among all of the known multiferroic materials, the $\mathrm{TbMnO}_{3}$ manganite has attracted considerable scientific attention in the last years due to strong coupling between ferroelectricity and magnetism [1,2]. The magnetoelectric and magnetocapacitive effects observed in the $\mathrm{TbMnO}_{3}$ ferroelectric can be used in novel magnetoelectric devices, in which the magnetic properties are electrically controlled and vice versa.

In several works [2-5] the structural, electric, thermal, and magnetic properties of the $\mathrm{TbMnO}_{3}$ single crystals as a function of temperature and magnetic field have been studied. According to a specific heat and neutron diffraction study of the $\mathrm{TbMnO}_{3}$ single crystal, the anomalies observed at 7 and $41 \mathrm{~K}$ are attributed to the antiferromagnetic ordering of the $\mathrm{Tb}^{3+}$ and $\mathrm{Mn}^{3+}$ moments, respectively. Mn spins order in a sinusoidal incommensurate structure. A spontaneous electrical polarization in the single crystal samples was observed below $28 \mathrm{~K}$, when the magnetic structure transforms from a sinusoidal incommensurate to a spiral antiferromagnetic one that testifies about very strong magnetoelectric coupling.

In works $[6,7]$ the magnetic properties of the $\mathrm{TbMnO}_{3}$ thin films have been studied. Neutron scattering measurements [7] have shown the presence of an intrinsic ferromagnetic ordering in an antiferromagnetic multiferroic $\mathrm{TbMnO}_{3}$ film at low temperatures. Since this antiferromagnet is piezomagnetic, the epitaxial strain was shown to induce a ferromagnetic moment in this film [6], whereas the electric polarization in the $\mathrm{TbMnO}_{3}$ films was not investigated.

In this paper, the measurements of ferroelectricity and magnetization of the $\mathrm{TbMnO}_{3}$ film have been performed that has allowed to observe the evolution of magnetic and electric properties of $\mathrm{TbMO}_{3}$ near the magnetic and

*corresponding author; e-mail: sazan@ifpan.edu.pl ferroelectric phase transitions as well as their control by the application of magnetic and/or electric fields.

\section{Preparation and measurement techniques}

The investigated $\mathrm{TbMnO}_{3}$ film, $150 \mathrm{~nm}$ thick, was grown using magnetron sputtering of target onto [100] $\mathrm{SrTiO}_{3}$ single crystal substrate in argon-oxygen gas atmosphere. The temperature of substrate was $650^{\circ} \mathrm{C}$ and deposition time was $3 \mathrm{~h}$.

Figure 1 presents X-ray diffraction structural analysis performed with low $(16 \mathrm{~h})$ and high $(2 \mathrm{~h})$ rate. It clearly shows diffraction peaks corresponding to cubic [100] oriented $\mathrm{SiTiO}_{3}$ substrate (at $2 \theta=22.8$ and $46.5^{\circ}$ ) and slightly shifted to the left orthorhombic [001] $\mathrm{TbMnO}_{3}$ film (at $2 \theta=23.8$ and $46.8^{\circ}$ ). The displacement of peaks corresponding to substrate and film could be justified by minor offset in stoichiometric composition of the film or mismatch of lattice parameters of the film and the substrate. Bulk $\mathrm{TbMnO}_{3}$ has the orthorhombic perovskite structure, which has lattice parameters of $0.393 \mathrm{~nm}$ in-plane and $0.370 \mathrm{~nm}$ out-of-plane [8], while the film has the perovskite structure with an out-of-plane lattice parameter of $0.372 \mathrm{~nm}$. Therefore, we conclude the film is compressively strained by the substrate resulting in the tetragonally distorted orthorhombic phase of $\mathrm{TbMnO}_{3}$. To find out the origin of the peak of $37.5^{\circ}$ the more detailed structural analysis must be performed.

The magnetic measurements were carried out using a commercial SQUID magnetometer in temperature range of $5-300 \mathrm{~K}$ in magnetic fields up to $5 \mathrm{~T}$.

Electric polarization was measured both at fixed magnetic field and fixed temperature using Keithley 6423 electrometer at temperatures of $5-45 \mathrm{~K}$ and in magnetic fields up to $8 \mathrm{~T}$. For measurements of the electric polarization, the silver contacts were plotted onto the widest faces of the rectangle $7 \times 3 \mathrm{~mm} \mathrm{TbMnO}_{3}$ film. Magnetic field was measured by conventional Hall sensor, temperature was controlled by thermometer mounted directly on insert connected to precision Lakeshore 340 temperature controller. The magnetocapacitance measurements have 


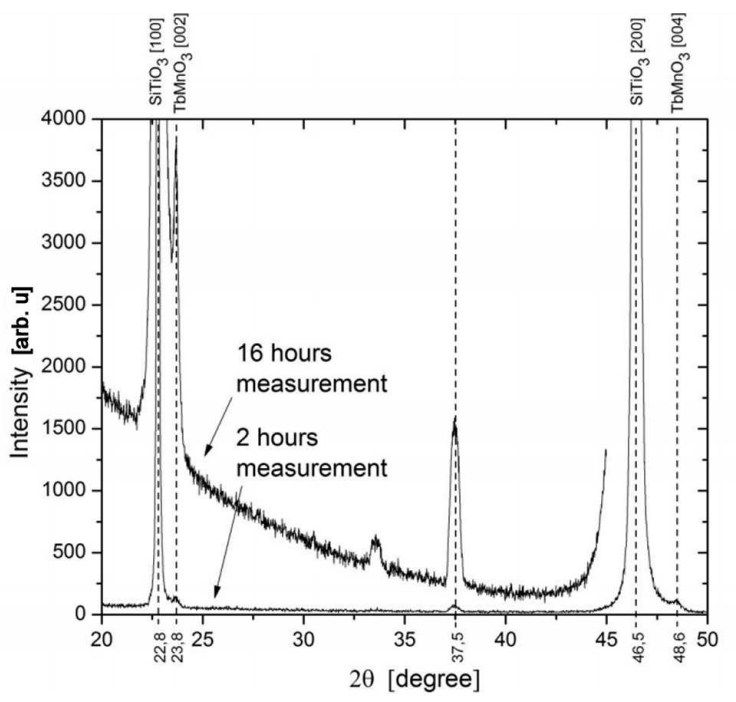

Fig. 1. X-ray diffraction spectra of $\mathrm{TbMnO}_{3} / \mathrm{SrTiO}_{3}$ film.

been performed by high precision bridge AH2550A with $1 \mathrm{kHz}$ frequency.

\section{Experimental results and discussion}

Temperature dependence of susceptibility, $M / H$, of the $\mathrm{TbMnO}_{3}$ film in magnetic field of $6 \mathrm{kOe}$ is presented in Fig. 2. The diamagnetic contribution arising from the substrate measured in a separate experiment was subtracted from the raw magnetization. The $M / H$ dependence shows a distinct anomaly at $45 \mathrm{~K}$. This anomaly is close to the anomalies of $C(T)$ and $M(T)$ observed in the $\mathrm{TbMnO}_{3}$ single crystal [2], which correspond to the magnetic phase transitions in $\mathrm{Mn}^{3+}$ sublattice.

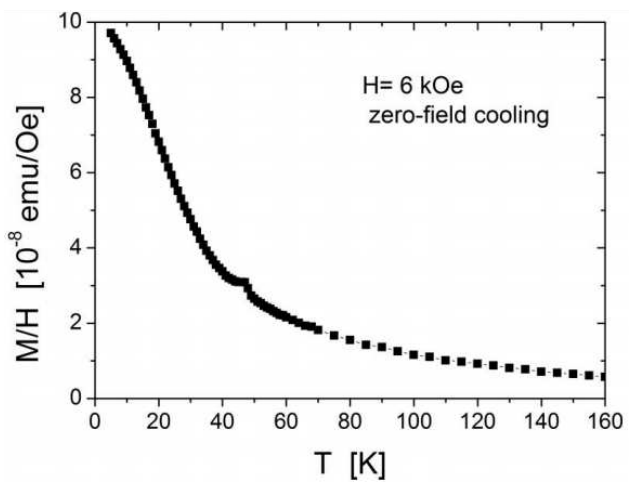

Fig. 2. Temperature dependence of $M / H$ for the $\mathrm{TbMnO}_{3}$ film with an anomaly near $45 \mathrm{~K}$.

However, the $M / H(T)$ dependence did not detect the anomaly observed at $27 \mathrm{~K}$ in the single crystal [2] and connected with an occurrence of the ferroelectric phase, followed by an incommensurate to commensurate magnetic phase transition in the Mn sublattice.

The hysteretic behavior in the $\mathrm{TbMnO}_{3}$ film was observed at low temperatures in the field range of $\pm 20 \mathrm{kOe}$. These measurements show that an antiferromagnetic

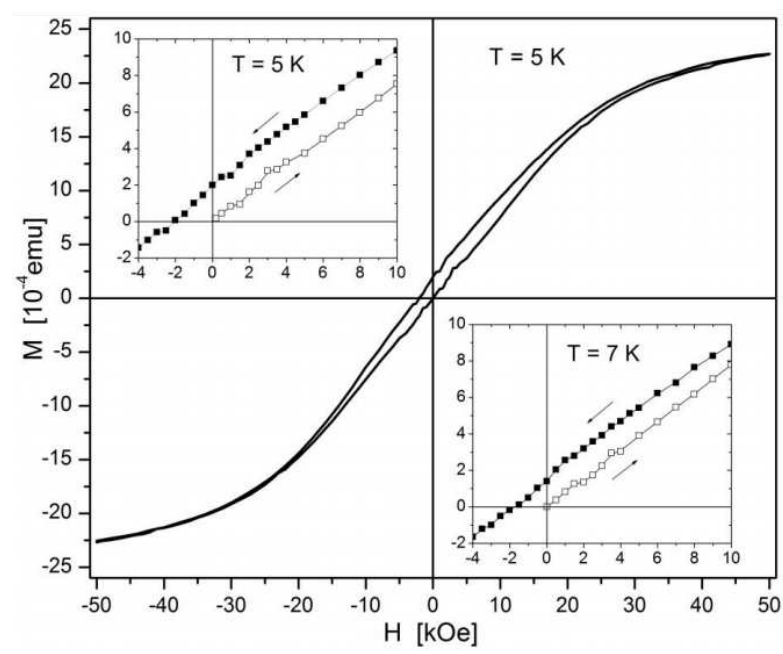

Fig. 3. Hysteresis loops at $5 \mathrm{~K}$ and $7 \mathrm{~K}$.

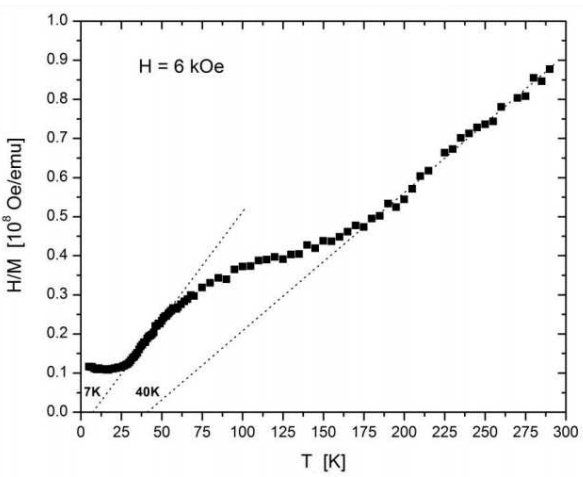

Fig. 4. Temperature dependence of reciprocal $H / M$ susceptibility for the $\mathrm{TbMnO}_{3}$ film in magnetic field of $6 \mathrm{kOe}$

multiferroic $\mathrm{TbMnO}_{3}$ film exhibits an intrinsic ferromagnetic order at low temperatures (Fig. 3).

This is in agreement with the polarized neutron reflectometry measurements data [7]. One of possible origin of the observed ferromagnetism in film is presumed to be the coupling between magnetization and film strain imposed by the substrate [6].

The measurements of temperature dependence of reciprocal susceptibility display the paramagnetic CurieWeiss temperatures equal to about $7 \mathrm{~K}$ and $40 \mathrm{~K}$ for $\mathrm{Tb}^{3+}$ and $\mathrm{Mn}^{3+}$ ions, respectively (Fig. 4).

The electric polarization of the $\mathrm{TbMnO}_{3}$ film as a function of temperature at different magnetic fields applied along the $a$ (in-plane) and $c$ (out-of-plane) configurations is presented in Fig. 5. Magnetoelectric measurements of $\mathrm{SrTiO}_{3}$ substrate were performed in a separate experiment, and the contribution arising from the substrate was subtracted from the raw polarization. It is seen that the electric polarization appears below $30 \mathrm{~K}$ in applied magnetic field of $H>1 \mathrm{~T}$ that is related to the occurrence of the ferroelectric phase.

There is no more difference between ferroelectric signal and noise of substrate above $35 \mathrm{~K}$. It is seen that 


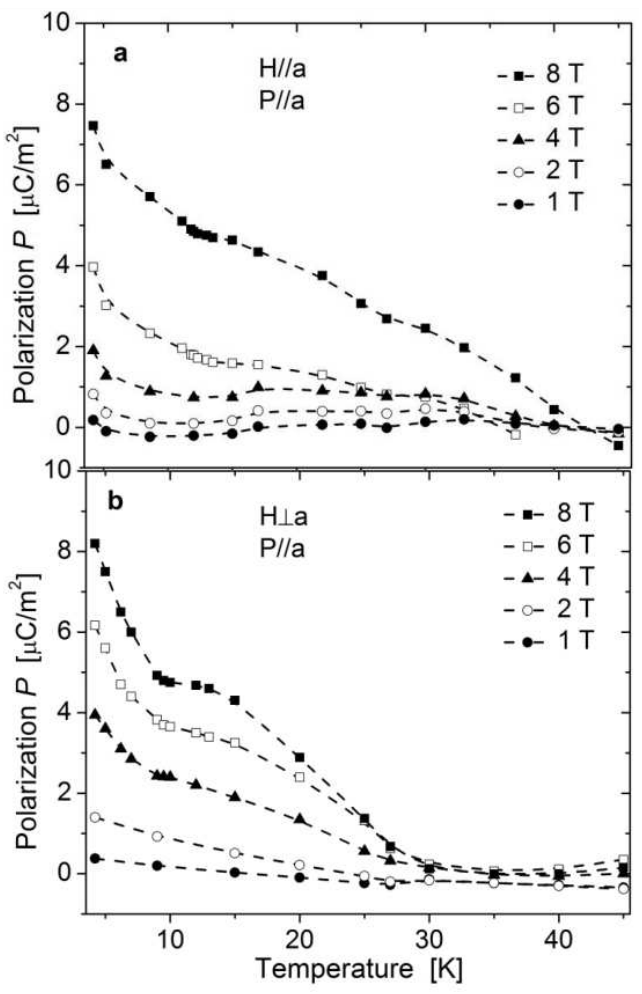

Fig. 5. Experimental electric polarization $P$ vs. temperature at various magnetic fields applied along the in-plane (a) and out-of-plane (b) of the sample.

the electric polarization depends strongly on both value and direction of magnetic field relative to the crystallographic axes. The electric polarization value increases with decreasing temperature and as magnetic field is increased. The onset temperature of ferroelectric ordering $\left(T_{\mathrm{FE}}\right)$ in magnetic field $H \perp a$ (Fig. $5 \mathrm{~b}$ ) is almost independent of field in contrast to $T_{\mathrm{FE}}$ in magnetic field applied along the $a$ axis (Fig. 5a) which shifts towards high temperatures with increasing magnetic field. The magnetic-field-induced electric polarization in magnetic field $H \perp a$ exceeds one for magnetic field applied along the $a$ axis. The significant increase of polarization is observed below $7 \mathrm{~K}$, especially for $H \perp a$ direction (Fig. 5). This data are in line with the measurements of dielectric properties (Fig. 4), where the saturation appears below $10 \mathrm{~K}$.

We have also performed measurements of the dielectric constant at $1 \mathrm{kHz}$ frequency as a function of temperature at magnetic fields applied perpendicular to the film plane.

Figure 6 presents the temperature variation of the dielectric constant, $\Delta \varepsilon / \varepsilon(0)$, in magnetic fields of 1 and $8 \mathrm{~T}$ applied perpendicular to the film plane. The dielectric constant value starts to be observed below $30 \mathrm{~K}$ that clearly shows the occurrence of ferroelectric ordering. The dielectric constant saturation below $10 \mathrm{~K}$ corresponds to an antiferromagnetic ordering of $\mathrm{Tb}^{3+}$ ions. The $\Delta \varepsilon / \varepsilon(T)$ behavior shows a slight magnetic field effect. These results are in agreement with early obtained data for the single crystal [4].

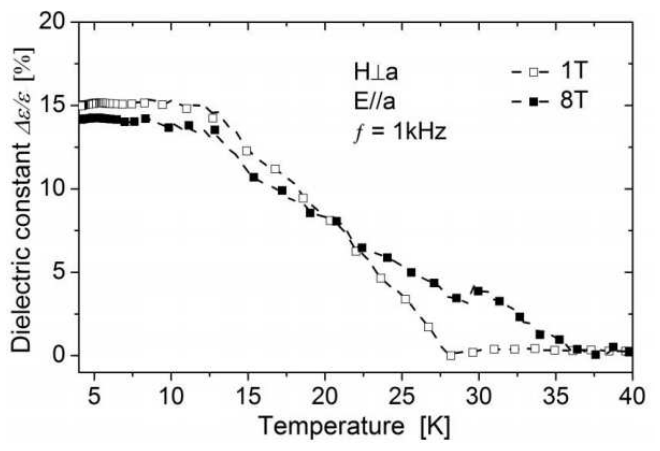

Fig. 6. Changes of dielectric constant, $\Delta \varepsilon / \varepsilon(0)$, vs. temperature at magnetic fields of 1 and $8 \mathrm{~T}$ for out-of-plane configuration.

\section{Conclusions}

In this paper, susceptibility and electric polarization measurements for the $\mathrm{TbMnO}_{3}$ film have been carried out. The magnetic and ferroelectric phase transitions were determined from the anomalies of magnetic and electric properties as a function of temperature, respectively.

Magnetic field dependences of magnetization show a manifestation of the low temperature ferromagnetism in the antiferromagnetic $\mathrm{TbMnO}_{3}$ film grown on [001] $\mathrm{SrTiO}_{3}$ substrate. However, they do not display signs of a phase transition related to the onset of ferroelectricity observed in the $\mathrm{TbMnO}_{3}$ single crystal. The measurements of temperature dependences of both the electric polarization and dielectric constant of the $\mathrm{TbMnO}_{3}$ film have been performed. It has been found that the charge ordering and ferroelectric phase appears below $30 \mathrm{~K}$ and significantly raises coming through $10 \mathrm{~K}$.

It should be noted that the temperature of ferroelectric phase transition slightly shifts towards high temperatures with applying or changing the direction of magnetic field.

\section{References}

[1] M. Fiebig, J. Phys. D 38, R123 (2005).

[2] T. Kimura, T. Goto, H. Shitani, K. Ishizaka, T. Arima, Y. Tokura, Nature 426, 55 (2003).

[3] T. Goto, T. Kimura, G. Lawes, A.P. Ramirez, Y. Tokura, Phys. Rev. Lett. 92, 257201 (2004).

[4] T. Kimura, G. Lawes, T. Goto, Y. Tokura, A.P. Ramirez, Phys. Rev. B 71, 224425 (2005).

[5] M. Kenzelmann, A.B. Harris, S. Jonas, C. Broholm, J. Schefer, S.B. Kim, C.L. Hang, S.-W. Cheong, O.P. Vajk, J.W. Lynn, Phys. Rev. Lett. 95, 087206 (2005).

[6] D. Rubi, C. de Graaf, C.J.M. Daumont, D. Mannix, R. Broer, B. Noheda, Phys. Rev. B 79, 014416 (2008).

[7] B.J. Kirby, D. Kan, A. Luykx, M. Murakami, D. Kundaliya, I. Takeuchi, J. Appl. Phys. 105, 07D917 (2009).

[8] J.A. Alonso, M.J. Martinez-Lope, M.T. Casais, M.T. Fernandez-Diaz, Inorg. Chem. 39, 917 (2000). 